\title{
A Comparative Study of Liver Function Test in a Group of Persons with Alcohol Dependence Disorder
}

Anumita Pushilal, Kamal Narayan Kalita*

Dept of Biochemistry, LGBRIMH, ${ }^{*}$ Dept of Psychiatry, LGBRIMH, Tezpur, Assam

\section{ABSTRACT}

Alcohol related diseases affect $5-10 \%$ of the world's population each year. Medical illness is a common consequence of heavy drinking. Chronic and excessive alcohol ingestion is one of the major causes of liver diseases. Laboratory testing can assist the clinician in providing objective, non-judgmental feedback to alcoholic patients on negative physical consequences of excessive drinking. Laboratory testing can also assist the clinician in providing objective, non-judgmental feedback to alcoholic patients on negative physical consequences of excessive drinking. This purposive cross sectional study aims to compare the values of hepatic enzymes along with total protein in a group of chronic alcoholics and a matched controlled group. 25 individuals fulfilling ICD-10 criteria for alcohol dependence syndrome were recruited and were applied AUDIT. The values of the hepatic enzymes were significantly raised along with reversal of the albumin globulin ratio in majority of the cases. Multiple physical problems were also observed in the study group.

Key Words : alcohol dependence, AUDIT, Liver function test, fatty liver, cirrhosis

Table 1. Global Burden of Disease attributable to Alcohol and other Drugs

\begin{tabular}{|llllllll|}
\hline Region & $\begin{array}{c}\text { Deaths } \\
(1000 \text { 's })\end{array}$ & $\begin{array}{c}\text { \%of } \\
\text { total } \\
\text { deaths }\end{array}$ & $\begin{array}{c}\text { Years } \\
\text { of } \\
\text { life lost } \\
(1000 \text { 's })\end{array}$ & $\begin{array}{c}\text { \%of } \\
\text { total } \\
\text { years of } \\
\text { life lost }\end{array}$ & $\begin{array}{c}\text { Years of } \\
\text { life } \\
\text { disabled } \\
(1000 \text { 's })\end{array}$ & $\begin{array}{c}\% \text { of } \\
\text { total } \\
\text { years of } \\
\text { life } \\
\text { disabled }\end{array}$ & $\begin{array}{c}\text { Disi } \\
\text { Adj } \\
\text { life } \\
\text { (DA } \\
(10\end{array}$ \\
\hline World & 773.6 & 1.5 & 19287 & 2.1 & 28400 & 6.0 & 476 \\
INDIA & 112.9 & 1.2 & 2723 & 1.4 & 1974 & 2.3 & 469 \\
Established & 83.8 & 1.2 & 2537 & 5.1 & 7667 & 15.6 & 102 \\
Market & & & & & & & \\
\hline
\end{tabular}

Economies

Correspondence : Dr. A Pushilal, Department of Biochemistry, LGB Regional Institute of Mental Health, Tezpur, Assam, 784001. E-mail: anumita_2007@rediffmail.com

\section{INTRODUCTION}

According to the 1993 World Development Report, alcohol related diseases affect 5-10\% of the world's population each year, and accounts for approximately $3 \%$ of the global burden of disease in 1990(Desjarlais et al 1995). The global burden of disease and injury attributable to alcohol use in 1990 are summarised in tablel (Murray and Lopez 1996).

Although the data represented in the table is less alarming than the global problem of alcohol that exists elsewhere, India has been seeing an increase in alcohol related problems. Our country has limitations in proper and uniform data in different aspects of alcohol use. Premrajan et al 1993 reported of prevalence of alcohol dependence disorder in 66.2 per thousand male in our country in an urban population.

Medical illness is a common consequence of heavy drinking ${ }^{1}$. No organ is immune to the effect of alcohol. In central nervous system, long term alcohol use can cause cognitive disturbances, sleep apnoea, REM sleep disorders, Warnicke-Korsakoff's syndrome, cerebellar degeneration, peripheral neuropathy and emotional problems. Alcohol causes oesophagitis, gastritis, pancreatitis and G.I bleeding. Mallory Weiss Syndrome is particularly important. Alcohol increases risk of cancers, particularly GI cancers, pancreatic cancer and breast cancer. Anaemia, increase in mean corpuscular volume (MCV), reticulocytopenia and hyperplastic bone marrow has been associated with chronic alcohol misuse. Heavy drinkers have increased risk of hypertension and chronic heavy drinkers are reported to have six-fold increased risk for coronary artery disease as well as increased risk of cardiomyopathy. Holiday Heart syndrome and different types of arrhythmias are reported to be associated with alcoholism. Chronic alcohol use has been reported to be related with testicular atrophy in males and decrease in ovarian size along with infertility in case of females. Alcohol use during pregnancy may lead to 
Fetal Alcohol Syndrome. Repeated heavy drinking is seen leading to change in calcium metabolism, low bone density, osteonecrosis, decrease in growth of epiphysis and thus increased risk of fractures. Persistent cortisolemia and change in vasopressin secretion keeps the alcoholics slightly over-hydrated. Moreover thyroid hormone levels are also altered. ${ }^{2}$

Arif and Westermeyer 1988 reported of some non specific health problems like malnutrition, muscle wasting, neuritis, vitamin deficiencies, infectious diseases (e.g. tuberculosis,dermatitis, pediculosis and hepatitis) and trauma secondary to fights and accidents associated with alcoholism.

Chronic and excessive alcohol ingestion is one of the major causes of liver disease. The pathology of alcoholic liver injury comprises three major lesions rarely existing in a pure form: (i) Fatty liver (ii) Alcoholic hepatitis (iii) Cirrhosis. Fatty liver is present in over $90 \%$ of binge and chronic drinkers. A much smaller percentage of heavy drinkers will progress to alcoholic hepatitis, thought to be a precursor to cirrhosis. The prognosis of severe alcoholic liver disease is dismal; the mortality of patient with alcoholic hepatitis concurrent with cirrhosis is nearly $60 \%$ at 4 years. Although alcohol is a direct hepatotoxin only $10-20 \%$ develops alcoholic hepatitis. The explanation for this paradox is unclear but involves the interaction of facilitating and co-morbid factors such as gender, heredity, infection and immunity etc. ${ }^{3}$

Alcohol consumption over 80 gms per day in male and 40 gms per day in female for more than 10 years have a significant risk for developing cirrhosis. ${ }^{4}$

\section{Table 2. Alcohol Equivalent}

$\begin{array}{lll}\text { Whisky } & 30 \mathrm{ml} & 10 \mathrm{gms} \\ \text { Wine } & 100 \mathrm{ml} & 10 \mathrm{gms} \\ \text { Beer } & 250 \mathrm{ml} & 10 \mathrm{gms}\end{array}$

(Source: Sherlock S, Dooley J, Diseases of The Liver and Biliary System, $9^{\text {th }} \mathrm{Ed}$. Pg 371.)

Alcohol can neither be stored nor excreted and hence has to be metabolised. A healthy individual cannot metabolise more than 160-180 grams per day and one gram of alcohol provides seven calories which is of poor nutritional quality. ${ }^{5}$

The metabolism of alcohol occurs by three pathwaysAlcohol dehydrogenase (ADH) in cytosol, microsomal ethanol oxidising system (MEOS) involving the enzyme Cyp.2E1 and catalase in peroxisomes. ${ }^{6}$ During the oxidation of alcohol to acetaldehyde the NADH: NAD ratio is reversed. Accumulation of NADH inhibits neoglucogenesis by preventing oxidation of lactate to pyruvate. Consequently lactic acidosis and hypoglycemia are seen with excess alcohol use. Accumulation of NADH also stimulates fatty acid synthesis producing accumulation of tri-acylglycerol in hepatocytes resulting in fatty changes. Acetic acid produced from acetaldehyde with aldehyde dehydrogenase act as substrate for the above reaction. The change in redox state of liver inhibits protein synthesis and stimulates lipid peroxidation. ${ }^{7}$ The microsomal oxidising system converts NADP to NADP+ by oxidising alcohol to acetic acid. This produces free radicals. The catalase system is not significant but it may become significant if higher concentration of hydrogen peroxide becomes available through beta oxidation of fatty acids in peroxisome.

Patients experiencing alcohol related disorders are typically reluctant to seek help or tend to under report the frequency and intensity of their drinking. Diagnostic laboratory markers can help to detect individuals who deny or minimize their alcohol consumption. Laboratory testing can help to resolve diagnostic dilemmas among patients whose self report information and physical findings are inconclusive. $^{8}$

Laboratory testing can also assist the clinician in providing objective, non-judgmental feedback to alcoholic patients on negative physical consequences of excessive drinking. Graphical representation of the repeated laboratory findings help the patients comprehend better about their problem. These allows the patient to appreciate the declining and eventual stabilization of laboratory indices thereby enhancing his/her motivation to maintain sobriety. ${ }^{9}$ Laboratory tests can also help detecting relapse and thus can prevent the reinstatement of alcohol dependence. Moreover, they can be used to evaluate effectiveness of specific therapeutic interventions. ${ }^{8}$

Liver function tests are easy to perform and are used often to assess the impact of alcohol on liver. The various liver function tests can be grouped based on its principal functions are enumerated below -

(i) Tests based on EXCRETORY function : measurement of bile salt/bile pigment, urbilinogen, stercobilinogen in blood and urine.

(ii) Tests based on serum ENZYMES : determination of transaminases (AST/SGOT, ALT/SGPT), alkaline phosphatase, gamma glutamyl transpeptidase (GGTP), 5' - nucleotidase, lactate dehydrogenase, iso-citrate dehydrogenase etc.

(iii) Tests based on SYNTHETIC function : prothrombin time, albumin , globulin, alpha feto-protein , plasma fibrinogen etc.

(iv) Tests based on METABOLIC capacity: Glucose Tolerance tests, fructose tolerance tests, anti-pyrine breath tests, plasma amino acid, ammonia, glutamine 
in CSF etc.

(v) Tests based on DETOXIFICATION: hippuric acid synthetase tests.

The present study aims to compare the values of hepatic enzymes namely AST, ALT, Alkaline phosphatase, GGTP along with total protein in a group of chronic alcoholics and a matched controlled group. It also aims to explore into various aspects of nature of intake of alcohol along with determination of some specific somatic symptoms.

\section{MATERIALS AND METHOD}

This is a cross sectional study done at tertiary care hospital having the facility for deaddiction. The study sample comprised of 25 individuals fulfilling the inclusion criteria. These individuals fulfilled the criteria for alcohol dependence disorder as per ICD- 10 clinical guidelines.

\section{Inclusion criteria:}

1) A diagnosis of alcohol dependence disorder as per ICD - 10 guidelines.

2) A score of 15 or more and 13 or more in The Alcohol Use Disorder Identification Test (AUDIT) questionnaire in case of male and female respectively.

3) Patients treated as inpatient for deaddiction.

4) Informed consent.

\section{Exclusion criteria:}

1) Presence of co-morbid psychiatric conditions.

2) Presence of co-morbid substance misuse disorder other than alcohol.

3) Known chronic medical illness involving respiratory, cardiovascular, central nervous system which needs regular intake of medicine for last 6 months.

4) Any known malignant condition.

5) Persons receiving any medications for last 6 months regularly for any illness.

\section{Control Group :}

25 people of similar socio-demographic profile were selected with their consent. Secrecy about their identity kept intact. These people did not have any intake of alcohol during last 1 year. A careful screening was done to exclude any serious medical conditions with a semistructured proforma. Candidates fulfilling the exclusion criteria for the test group were exempted.

Purposive samples were collected on simple random basis. After clinical evaluation along with the sociodemographic profile, fasting blood samples were collected using standard aseptic measures using $5 \mathrm{ml}$ disposable syringes with hypodermic needles. The collected blood was kept in a stoppered vial for 30-45 minutes and allowed to clot. The serum thus collected was centrifuged at 3000 rpm for 3 minutes in a clinical centrifuge machine. The serum was used to measure alanine aminotransferase (ALT/SGPT), aspartate aminotransferase (AST/SGOT), total protein and fractions, total bilurubin and fractions, alkaline phosphatase, gamma-glutamyl transpeptidase/ transferase, fasting blood sugar, serum creatinine and blood urea using standard procedures. Value thus obtained were analysed using parametric statistical methods.

\section{Tools :}

1) A semi structured proforma for collecting sociodemographic variables.

2) A semi-structured schedule with points favouring the exclusion criteria and special enquiries in relation to alcohol use like duration of use, amount taken, type of beverage along with frequency and nature of intake.

3) ICD-10 criteria for clinical description and diagnosis guidelines: International classification of diseases and related health problems, $10^{\text {th }}$ revision is the current diagnostic guideline for diagnosing the health problems across the globe adopted by the World Health Organisation. The chapter V (F) is related to behavioural problems.

4) Alcohol Use Disorder Identification Test, AUDIT ${ }^{10}$ : It is a 10 item screening tool. It covers the domain of alcohol consumption, symptoms of alcohol dependence and alcohol related consequences. A score of 8 or more is associated with harmful or hazardous drinking, a score of 13 or more in women, and 15 or more in men is likely to indicate alcohol dependence. It has been shown to be sensitive and specific. It has been found superior to Michigan Alcohol Screening Test (MAST) in identifying hazardous drinkers, that is, those heavy drinkers who have not yet experienced serious harm from their drinking. ${ }^{11}$

\section{RESULTS}

Both the Study and Control groups contain 25 persons. The Socio-Demographic characteristics are shown in Table3. They are matched appropriately. The mean age of the two groups do not differ significantly(Table 4 ). $84 \%$ of the patients with alcohol dependence had poor appetite, while $60 \%$ had decreased sleep. Generalized weakness is also a very common feature (Table 5).

Fasting blood sugar level, serum creatinine and blood urea in the two groups were comparable and no significant association was observed with intake of alcohol with these parameters(Table 6).

The mean value of AST, ALT, ALP differed significantly in the said two groups. The persons with alcohol dependence had higher values of the hepatic enzymes, and significant associations were observed. The mean total protein in the study and control groups was $6.73 \pm 0.14$ and $7.0 \pm 0.85$ respectively. The persons with alcohol dependence had significantly lower value of serum albumin. The mean GGT value in the study group was 
Table 3. Socio-Demographic Variables

Study group Control Group

\begin{tabular}{llcc} 
Sex & Male & 23 & 21 \\
\multirow{5}{*}{ Locality } & Female & 2 & 4 \\
& Rural & 11 & 10 \\
& Urban & 14 & 15 \\
Education & Primary & 4 & 3 \\
& High School & 9 & 7 \\
& Matriculate & 5 & 5 \\
& Graduate & 7 & 10 \\
& Low & 3 & 2 \\
& Lower middle & 11 & 9 \\
& Upper middle & 8 & 8 \\
Occupation & High & 3 & 6 \\
& Unskilled & 5 & 2 \\
& Skilled & 5 & 7 \\
& Job & 10 & 10 \\
& Self-employed & 5 & 6 \\
Family type & Nuclear & 18 & 20 \\
& Joint & 7 & 5
\end{tabular}

$322.48 \pm 48.89$ (Table 7), and $68 \%$ had AST: ALT ratio more than 1 ( figure 1).

In the study group, we got mean alcohol intake per day $89.48 \mathrm{gms} /$ day $\pm 5.54 \mathrm{gms} /$ day and a mean year of use of alcohol was $19.2 \pm 1.47$ year. $36 \%$ of the cases took country liquor while $32 \%$ each took foreign liquor and mixed type.

\section{DISCUSSION}

Of the 25 persons with alcohol dependence disorder, $8 \%$ were female. Lesieur et al 1993 reported that women are more likely to abstain from alcohol, and if they do drink, they are more likely to consume lesser amount. It may be due to cultural factors along with some special biological factors. It is comparable to a recent study. ${ }^{12} 56 \%$ of the persons were found from urban background whereas $84 \%$ had education more than primary education. $48 \%$ of the persons who sought for deaddiction were matriculate. Thus the problem of Alcohol Dependence Disorder is widespread and is of similar distribution among rural and urban population. ${ }^{12}$ Education may be a factor related for seeking treatment in case of a person with alcohol dependence disorder. In our study we did not find any relation between habit of taking alcohol and occupation. We got $56 \%$ of the persons were from lower socioeconomic status. But the difference was not significant.

Table 4. Age Distribution

Study group

$(\mathrm{N}-25)$

Age Group

Frequency

(Mean in yrs)

21- 30

4

6

13

$(44.04 \pm 1.89)$

$51-60$

1

$61-70$

$$
\mathrm{t}=0.86 \quad \mathrm{df}=48
$$

1

$\mathrm{p}>0.05$

Frequency

\section{3}

5

11

5

3

5

Control Group

$(\mathrm{N}-25)$

(Mean in yrs)

$(41.84 \pm 1.74)$

$\pm 1.74)$

$>0.05$

Table 5. Special Symptoms and signs in the Study Group

SYMPTOMS No.of subjects Percentage

Loss of Weight $\quad 8 \quad 32$

Loss of Appetite $\quad 21 \quad 84$

Decreased Sleep $\quad 15 \quad 60$

Jaundice/Icterus $\quad 9 \quad 36$

$\begin{array}{lll}\text { GI Bleed } & 2 & 8\end{array}$

$\begin{array}{lll}\text { Loss Of Libido } & 10 & 40\end{array}$

$\begin{array}{lll}\text { Generalised Weakness } & 17 & 68\end{array}$

$\begin{array}{lll}\text { Swelling of Legs } & 6 & 24\end{array}$

Swelling Of Abdomen $\quad 5 \quad 20$

Pain Abdomen $\quad 10 \quad 40$

$\begin{array}{lll}\text { Anaemia } & 9 & 36\end{array}$

Hepatomegaly $\quad 8 \quad 32$

In a recent study conducted by WHO in collaboration with NIMHANS in Karnataka, rural areas with tribal population were found to have higher prevalence ${ }^{12}$. Since it is a hospital based study, the differences can be understood. $72 \%$ subjects were from nuclear family. It is an interesting finding. Due to westernization of Indian society, the joint families have broken up. So the new set of family norms have set up in modern India and old cultural values are being ignored day by day. Respect for seniors and fear of dishonour from juniors in a joint family may act as protective factors against alcohol taking 
Table 6. Serum sugar, creatinine and blood urea in both groups

$\begin{array}{llll}\begin{array}{l}\text { Study Gr } \\ \text { (Mean) }\end{array} & \begin{array}{l}\text { Control Gr } \\ \text { (Mean) }\end{array} & \text { t df } & \text { p }\end{array}$

$\begin{array}{llllll}\text { FBS } & 91.72 \pm 2.44 & 97.4 \pm 2.73 & 1.55 & 48 & \mathrm{p}>0.05\end{array}$

$\begin{array}{llllll}\text { Serum } & 1.7 \pm 0.2 & 0.92 \pm 0.03 & 1.3 & 48 & \mathrm{p}>0.05\end{array}$

Creatinine

$\begin{array}{llllll}\text { Blood } & 29.52 \pm 3.02 & 28 \pm 1.17 & 0.47 & 48 & \mathrm{p}>0.05 \\ \text { Urea } & & & & & \end{array}$

Table 7. Liver function parameters in both groups

\begin{tabular}{|c|c|c|c|c|c|}
\hline Parameters & Study & Control & $\mathrm{t}$ & df & $\mathrm{p}$ \\
\hline ALT & $122.88 \pm 33.9$ & $33.72 \pm 1.8$ & 2.67 & 48 & $\mathrm{p}<0.05$ \\
\hline $\mathrm{AST}$ & $187.16 \pm 73.1$ & $30 \pm 1.5$ & 2.14 & 48 & $\mathrm{p}<0.05$ \\
\hline Total Protein & $6.73 \pm 0.24$ & $7.0 \pm 0.85$ & 1.67 & 48 & $p>0.05$ \\
\hline Albumin & $3.22 \pm 0.17$ & $4.08 \pm 0.08$ & 4.49 & 48 & $\mathrm{p}<0.001$ \\
\hline \multicolumn{2}{|c|}{ Total Bilirubin $1.33 \pm 0.2$} & $0.59 \pm 0.05$ & 3.52 & 48 & $\mathrm{p}<0.01$ \\
\hline \multicolumn{2}{|c|}{ Direct Bilirubin $0.47 \pm 0.09$} & $0.23 \pm 0.04$ & 2.38 & 48 & $\mathrm{p}<0.05$ \\
\hline \multicolumn{6}{|l|}{ Alkaline } \\
\hline Phosphatase & $178.92 \pm 15.57$ & $84.48 \pm 2.58$ & 5.98 & 48 & $\mathrm{p}<0.001$ \\
\hline GGT & $322.48 \pm 48.89$ & $39.6 \pm 1.82$ & 5.78 & 48 & $\mathrm{p}<0.001$ \\
\hline
\end{tabular}

behaviour.

Global Status Report on Alcohol Reports that the per capita consumption of alcohol in India is 0.2litres per person per year (WHO 1999). But the recent study in Karnataka by WHO in collaboration with NIMHANS reports a much higher value as it has also taken the undocumented beverages under account. In the present study we got mean alcohol intake per day to be $89.48 \mathrm{gm} /$ day. This is a rough value as it is difficult to standardise the local alcoholic beverages. But an intake of alcohol more than 100gms/ day has been associated with cirrhosis of liver ${ }^{13}$.Country liquors were used more than the Indian made foreign liquors in the present study. It is consistent with other findings ${ }^{12}$. This may be due to lower price and wider availability. The mean duration of intake for the study group is 19.2 years. It is a significant finding as it shows that the persons are developing alcohol dependence at an earlier age as compared with earlier studies ${ }^{14}$. People have started to consume alcohol in their early twenties ${ }^{12}$. $84 \%$ subjects complained of loss of appetite while only 8
$\%$ reported of gastrointestinal bleeding. $20 \%$ subjects had ascites. $40 \%$ had complained of abdominal pain and also loss of libido. High existence of physical problems bears a significant cost. Symptoms like poor sexual performance may lead to marital disharmony and it may have psychological consequences in the spouse. In a deaddiction programme due importance to the physical problems are of utmost importance as these have direct implication on motivation. In the survey done by WHO and NIMHANS, a very low prevalence of physical problem existed in the group of alcoholics. They have found significant relationship of heavy drinking style with abdominal pain, headache, pain syndromes and visual problems ${ }^{12}$. This may be explained by the design of the study and nature of the samples included in the respective studies. They have not found any significant relationship with the type of drink i.e IMFL and country liquor with these physical problems. That means all type of alcohol is physically hazardous.

In the current study we have not found any significant difference in the fasting blood sugar, serum creatinine and blood urea in both the groups. An increased level of serum creatinine, if present is an ominous sign, since it frequently predicts the onset of hepato-renal syndrome ${ }^{15}$. These

AST:ALT

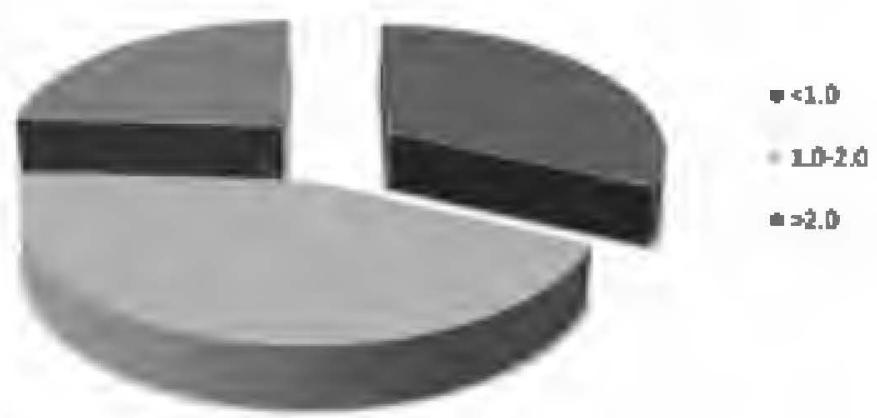

Figure 1. AST: ALT ratio

values are of importance for its therapeutic applications in deaddiction treatment as many drugs tend to have impact on these values. As we had excluded the chronic medical conditions from the study group, so normal values in these tests were expected.

The transaminase levels namely AST and ALT are increased significantly in the study group. Both the parameters passed the normality test in the two groups. ALT is more liver specific and rises in hepatic parenchymal disease. But in case of chronic alcoholic patient the relative rise in AST is higher than that of ALT. In our study we found AST: ALT ratio to be 0.89 and 1.59 in the control and test group respectively. From figure 1 , we get that 8 persons had a ratio of AST:ALT less than 1,11 patients had in between $1.0-2.0$ and 6 persons had a ratio of more than 2 in the test group. An AST level 
more than twice ALT level had been reported in as many as $83 \%$ of patients hospitalised for alcoholic hepatitis ${ }^{16,17}$. Zhon et al 1998 reported that ethanol significantly up regulates the expression and export of AST in Hep G2 cells ${ }^{18}$. Sorbi Et al 1999, in a study with 70 subjects each with alcoholic hepatitis and non-alcoholic steato-hepatitis (NASH) reported that a AST:ALT ratio of less than or equal to1 will correspond to a specificity and positive predictive value of $100 \%$ at an expense of a sensitivity of $80 \%$. While an increase in the ratio to 1.3 would maximise the sensitivity as well specificity and positive predictive value $^{19}$. Our finding goes in accordance. Depletion of pyridoxal -5-phosphate, is postulated to be the underlying mechanism for this increased AST:ALT ratio ${ }^{20,21}$.

The total protein of plasma is a mixture of simple proteins and conjugated proteins like glycoproteins, lipoproteins and immunoglobulins. All plasma proteins except immunoglobulins are synthesised in the liver. The serum albumin is an index of severity and prognosis in patients with chronic hepatic disease. In the present study, we got a mean serum protein of $6.73 \pm 0.14 \mathrm{gm} / \mathrm{dl}$ and $7.0 \pm 0.85$ $\mathrm{gm} / \mathrm{dl}$ in the test and control group respectively. In the control group the distribution was normal but in the test group it was skewed towards right. Hence in the present study we did not find any significant difference in the serum protein levels in the two groups but when normality tests is cleared for the test group, a significantly decreased mean value is found in the test group. Moreover the mean value of albumin in the two groups where $3.22 \pm 1.77 \&$ $4.08 \pm 0.08 \mathrm{gm} / \mathrm{dl}$ for the test and control group respectively. The difference was found to be statistically significant. The albumin and globulin ratio was 0.95 and 1.42 in the test and control group respectively. In the control group no sample had less than 1 ratio. So we get an alteration of the albumin globulin ratio in the test group. Similar findings have been reported in a study done by Dunn et al $2006^{22}$. They proposed a model for diagnosing alcoholic basis for steatohepatitis thus differentiating alcoholic liver disease from non-alcoholic steatohepatitis by an index called Alcoholic Non alcoholic steatohepatitis index (ANI). ${ }^{22}$

Bilirubin concentration can be increased in blood due to increased production, decreased uptake by liver, deceased conjugation, decreased secretion by liver or blockage of bile duct. In case of increased production, decreased uptake by liver or decreased conjugation, the unconjugated or indirect bilirubin is primarily elevated. In case of alcohol many mechanism may be implicated, for example, increased production due to megaloblastic anaemia, decreased conjugation due to primary liver disease or decreased secretion from narrowing of the bile canaliculi. The samples in the test group had significant higher mean value of both total bilirubin and its fraction in comparison to that of the samples in the control group. Similar increased value has been reported by Huang et al $1996^{23}$. They have found that interleukin $-8(\mathrm{IL}-8)$ has a weak positive correlation with the serum bilirubin level. High value of $I \mathrm{~L}-8$, IL- 6 and TNF-a may have poor prognostic value. ${ }^{23}$

Elevation in alkaline phosphatase level which originate primarily from two sources, liver and bone ${ }^{24}$. Elevated alkaline phosphatase level along with elevation of either of 5'-nucleotidase or gglutamyl transferase point towards a hepatobiliary origin ${ }^{25}$. In the present study we observed significant increase in alkaline phosphatase level in the test group as compared to that of control. Similar findings have been reported by Dunn et al $2006^{22}$. Due to lack of specificity there is relative scarcity of association of alkaline phosphatase level with alcoholic liver disease as compared with that of other hepatic enzymes.

Elevated levels of gglutamyl transferase levels are seen in wide variety of clinical conditions including hepatopancreato-biliary disease, myocardial infarction, renal failure, chronic obstructive pulmonary disease, diabetes and alcoholism ${ }^{26}$. It has been increased in persons taking anti-convulsants like phenytoin and barbiturates ${ }^{27}$. We observed a very significant rise in the level of GGT in the study. It goes in accordance of the previous findings. Results of the WHO/ISBRA collaborative project demonstrated that serum GGT is increased in $52 \%$ of alcohol dependant subjects, in $28 \%$ of heavy drinkers, in $15 \%$ of light/moderate drinkers and in $10 \%$ of nondrinkers ${ }^{28}$. Elevated GGT is an early indicator of liver disease. Heavy alcohol intake induces a rise in serum GGT levels that return to the reference range after 20-30 days of abstinence. Increased serum GGT is a sensitive marker $(60-90 \%)$ for the diagnosis of alcohol induced damage but only if associated with other markers of alcohol misuse and when biliary stasis is excluded ${ }^{29}$. Again Rosalki et al 1984 reported that GGT is more likely to be elevated in regular drinkers as compared to the episodic drinkers. Psychiatrist should be able to diagnose active hepatic pathology in the patients attending deaddiction clinics and should not hesitate to take help from the hepatologist whenever needed. The biochemical profile and the radiological evidences give us many clues about the hepatic status of the patient. Usually the combination of elevated AST (but <300 IU/ml) and AST:ALT>2, a total serum bilirubin level of more than $5 \mathrm{mg} / \mathrm{dl}$ ( $86 \mathrm{imol} / \mathrm{l})$, an elevated INR, and neutrophillia in a patient with ascites and history of heavy alcohol use is indicative of alcoholic hepatitis until proven otherwise. ${ }^{30}$ Various scoring systems have been used to assess the severity of alcoholic hepatitis to guide the treatment protocol. Maddreys Discriminant 
Function, The Glasgow Score and the score on Model for End Stage Liver Disease (MELD), help the clinician whether to initiate corticosteroid or not, whereas Lilles score help in deciding about termination of corticosteroid therapy after 1 week ${ }^{30}$. Forest et al 2007 reported that patients with a Maddreys Discriminant Function of 32 or more and a Glasgow alcoholic hepatitis score of 9 or more who were treated with corticosteroid had an 84 day survival rate of $59 \%$, as compared with $38 \%$ survival rate among untreated patients. ${ }^{31}$

Thus the present findings have significant implications in total management of a person with alcohol dependence. The main limitation of the present study is that it a crosssectional study having lower sample size. Moreover correlation of the different enzyme levels with the pattern of alcohol intake and the AUDIT score would have been more informative. Still our study has got the strength of extensive light on the biochemical profiles of the patients with alcohol dependence disorder.

\section{REFERENCES}

1. Baber F, Stephens RS, Marlatt GA (1987). Social drinking as a health and psychosocial risk factor: Anstie's limit revisited. In Recent Developments Of Alcoholism. Vol 5. Galanter M (ed). Plenum Press, New York, pp 373-402.

2. Marc A. Schuckit. Alcohol and Alcoholism. In Fauci SA, Kasper DL, Longo DL, Braunwald E, Hauser S, Jameson JL, Loscalzo J eds. Harrisons Principles Of Internal Medicine, $17^{\text {th }}$ ed. Mc Graw Hills. 2007: 2562-2564.

3. Mailliard ME, Sorel MF. Alcoholic Liver Disease. In Fauci SA, Kasper DL, Longo DL, Braunwald E, Hauser S, Jameson JL, Loscalzo $\mathrm{J}$ eds. Harrisons Principles Of Internal Medicine, $16^{\text {th }}$ ed. Mc Graw Hills. 2005: 1855-1856.

4. Florence Wong etal. Alcoholic Liver Disease: Medicine International( Indian ed) 1994. 469-471.

5. Sherlock S, Dooley J. Diseases of the Liver and Biliary System. $9^{\text {th }}$ ed. $\operatorname{Pg} 373$.

6. Maher JJ. Exploring alcohol's effect on liver function. Alcohol Health and Research World. 1997; 21: 5-12.

7. Situnayake RD, Crump BJ, Thurnham DI etal. Lipid peroxidation and hepatic antioxidants in alcoholic liver disease. Gut 1990; 31: 1311-1313.

8. Allen JP, Litten R. The role of laboratory tests in alcoholism treatment. J Substance Abuse Treatment. 2001; 20: 81-85.

9. Miller WR, Zweben A, Diclemente CE et al. Motivational Enhancement Theory Manual:A Clinical Research Guide For Therapist Treating Individuals with Alcohol Abuse and Dependence. 1999. Project MATCH Monograph series, vol. 2, Publication number 94- 3723. National Institute on Alcohol Abuse and Alcoholism, Rockville MD.

10. Saunders JB, Aasland OG, Babor TF etal. Development of the alcohol use disorders identification test (AUDIT): WHO collaborative project on early detection of persons with harmful alcohol
consumption-II. Addictions. 1993; 88: 791-804.

11. Bohn MJ, Babor TF, Kranzler HR. the alcohol use disorder test (AUDIT): validation of the screening instrument for use in medical settings. J Stud Alcohol. 1995; 56: 423-432.

12. WHO Collaborative Project on Unrecorded Consumption of Alcohol, Karnataka, India, 2007.

13. Bellantani S, Saccaccio G, Costa G et al. Drinking habits as co-factor of risk for alcohol induced liver damage. Gut 1997; 41: 845-50.

14. Benegal V. Dimensions of Substance Abuse Problems and the Need to Network . Deaddiction Quarterly. 1998; 2 and 3: 3-6.

15. Multimer DJ, Burra P, Neuberger JM et al. Managing Severe Alcoholic Hepatitis Complicated by Renal Failure. Q J Med. 1993; 86: 649-56.

16. Pinto HC, Baptista A, Camilo ME et al. Non Alcoholic Steatohepatitis: Clinicopathological Comparison with Alcoholic Hepatitis in Ambulatory and Hospitalised Patients.Dig Dis Sci 1996; 41: 172-9.

17. Bird GLA. Investigation of Alcoholic Liver Disease. Bailliere's Clinical Gastroenterology 1993; 7: 663-82.

18. Zhon SL, Gorden RE, Bradbury $M$ et al. Ethanol upregulates fatty acid uptake and plasma membrane expression and export of aspartate aminotransferase in HepG2 cells. Hepatology 1998; 27: 1064-74.

19. Sorbi D, Boynton J, Lindor KD. The Ratio of Aspartate Aminotransferase to Alanine Aminotransferase: Potential Value in Differentiating Nonalcoholic Steatohepatitis from Alcoholic Liver Disease. The American J. of Gastroenterology. 1999; 94-4: 10181022.

20. Cohen et al. SGOT: SGPT ratio- an indicator of Alcoholic Liver Disease. Dig Dis Sci. 1979; $24: 835$.

21. Ludwig S, Kaplowitz N. Effect of pyridoxine deficiency on serum and liver transaminases in experimental liver injury in rat. Gastroenterology 1980; 79: 545-9.

22. Dunn W, Angnlo P, Sandersons et al. Utility of a New Model to Diagnose an Alcohol Basis for Steatohepatitis. Gastroenterology 2006; 131: 1057-1063.

23. Huang Y, Chan C, Wui J, Pai C,et al. Serum level of interlukin-8 in alcoholic liver disease, relationship with disease stage, biochemical parameters and survival. Journal of Hepatology. 1996; 24: 377-84.

24. Pratt DS, Kaplan MM. Laboratory Tests. In: Schiff ER, Sorell MF, Maddrey WC, eds. Sciffs Diseases of the Liver, $8^{\text {th }}$ ed. Vol.1.Philadelphia: Lippincott- Raven, 1999:205-44.

25. Pratt DS, Kaplan MM. Evaluation of abnormal liver enzyme results in asymptomatic patients. The New England J. of Medicine, 2000, 342:17:1266-71.

26. Goldberg, MartinJV, Role of Gamma Glutamyl Transpeptidase Activity in the Diagnosis of Hepatobiliary Disease. Digestion $1975 ; 12: 232-46$.

27. Rosalki SB, Tarlaw D, Ran D. Plasma Gamma Glutamyl Transpeptidase elevation in Patients Receiving Enzyme Inducing Drugs, Lancet 1971;2:376-7.

28. Helander A. Biological Markers in Alcoholism. J Neural Transm Suppl, 2003;66:15-32.

29. Mancinelli R, Ceccanti M. Biomarkers in Alcohol Misuse: Their role in the Prevention and Detection of Thiamine Deficiency, Alcohol and Alcoholism 2009;44-2:177-182.

30. Lucey MR, Mathurin P, Morgan TR. Alcoholic Hepatitis. The New England J of Medicine, 2009;360:2758-69.

31. Forrest EH, Morris AJ, Stewart $\mathrm{S}$ et al. The Glasgow Alcoholic Hepatitis Score Identifies Patients Who May Benefit From Corticosteroids. Gut 2007;56:1743-6. 